Silicon-photonics microwave oscillators closer to commercialisation

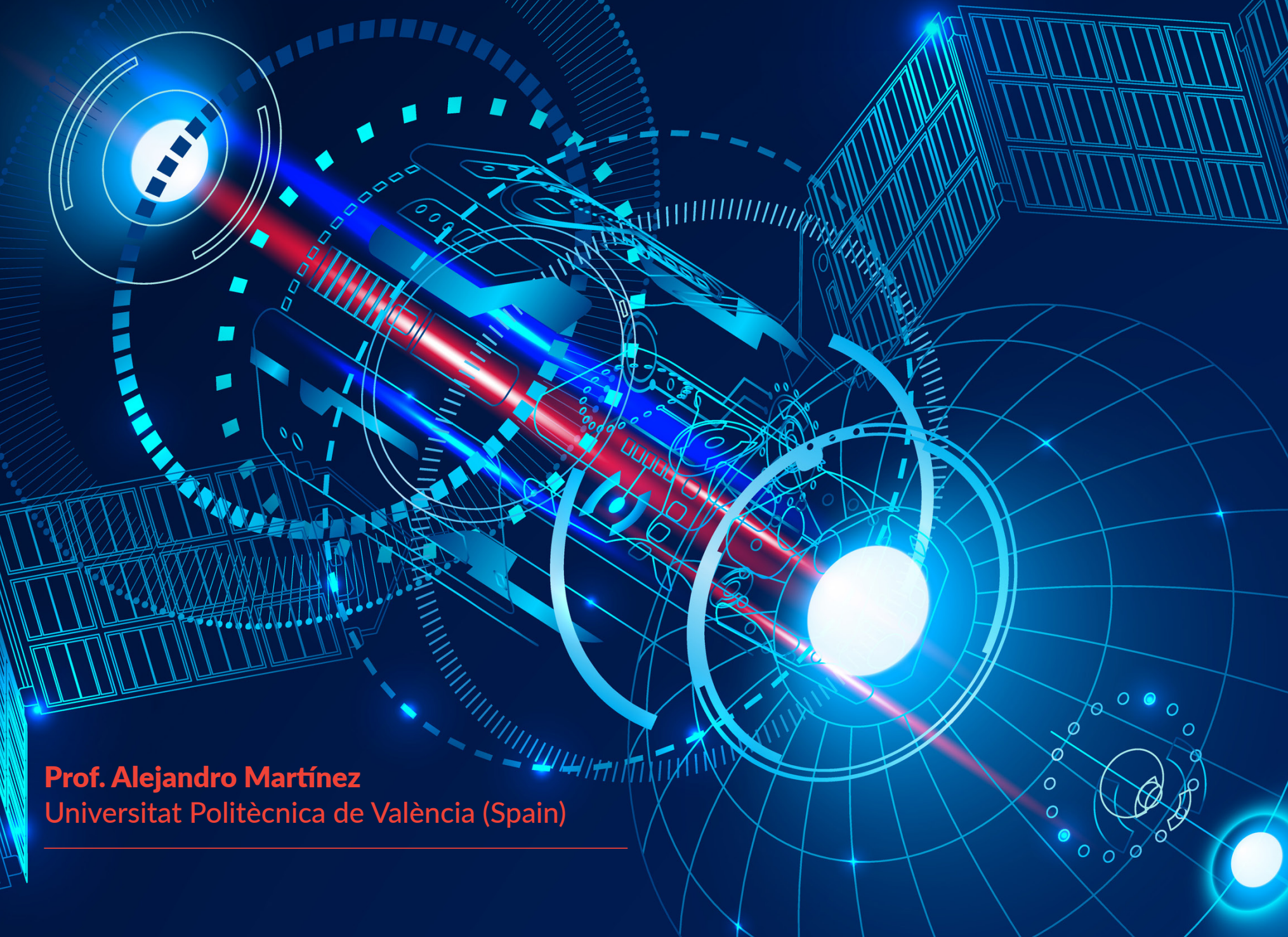

Microwave sources displaying high spectral purity are required in a myriad
of applications spanning radar, wireless networks, radioastronomy and satellite communications. Typically, low-noise communications. Typically, low-noise
microwave oscillators are made by microwave oscillators are made by
applying frequency multiplication to a applying frequency multiplication to requires a cascade of frequency-doublin requires a cascade of frequency-doubling
stages, which adds noise and reduces the stages, which
signal power.

Recently, techniques to produce microwave tones using photonics have been proposed. The resulting device tench optoelectronic oscilator (OEO) and it possesses several advantages with esper in. 1. 1 , compactness, long-distance transport using optical extremely-10w noise.

First Gealisations of OEOs included ong paths of optical fibre as a feedback mechanism to achieve oscillation. Thi resulted in bulky, heavy devices, not appropriate in applications requiring compactness and low weight. An meresting alternative to generate mestem system is va mechenical waves coexisting and interacting with optcal waves in serde of the OEO since the wavelength of f the ozo since the wave ength of mechenter waves in solds is about five orders of magnitude smaller than in ther

In the PHENOMEN project (funded by H2O2O under the FET-Open programme) researchers of the Nanophotonics Technology Center (NTC) in the Universitat Politècnica de València (UPV Spain) designed and demonstrated th an optomechanical cavity (Figure 1) with a foot-print of the order of several $\mu \mathrm{m}^{2}$ could perform as an ultra-compact OEO The cavity, which was built on a silicon chip using standard nanofabrication tools, supported confined optical and mechanical modes that could strong interact because they coexist in is propery ope device telecom-wavelength laser, mechanical vibrations inside the cavity are amplified, (n) osciltions (or phome lasing, which is frequency was $4 \mathrm{GHz}$, and the measured vase noise, the metric determining the waves instead of ight). Then, the laser purty of the signal, was below -100dBc multiples of thes, corresponding to tradtokal, bukcer solutions (Mercade et
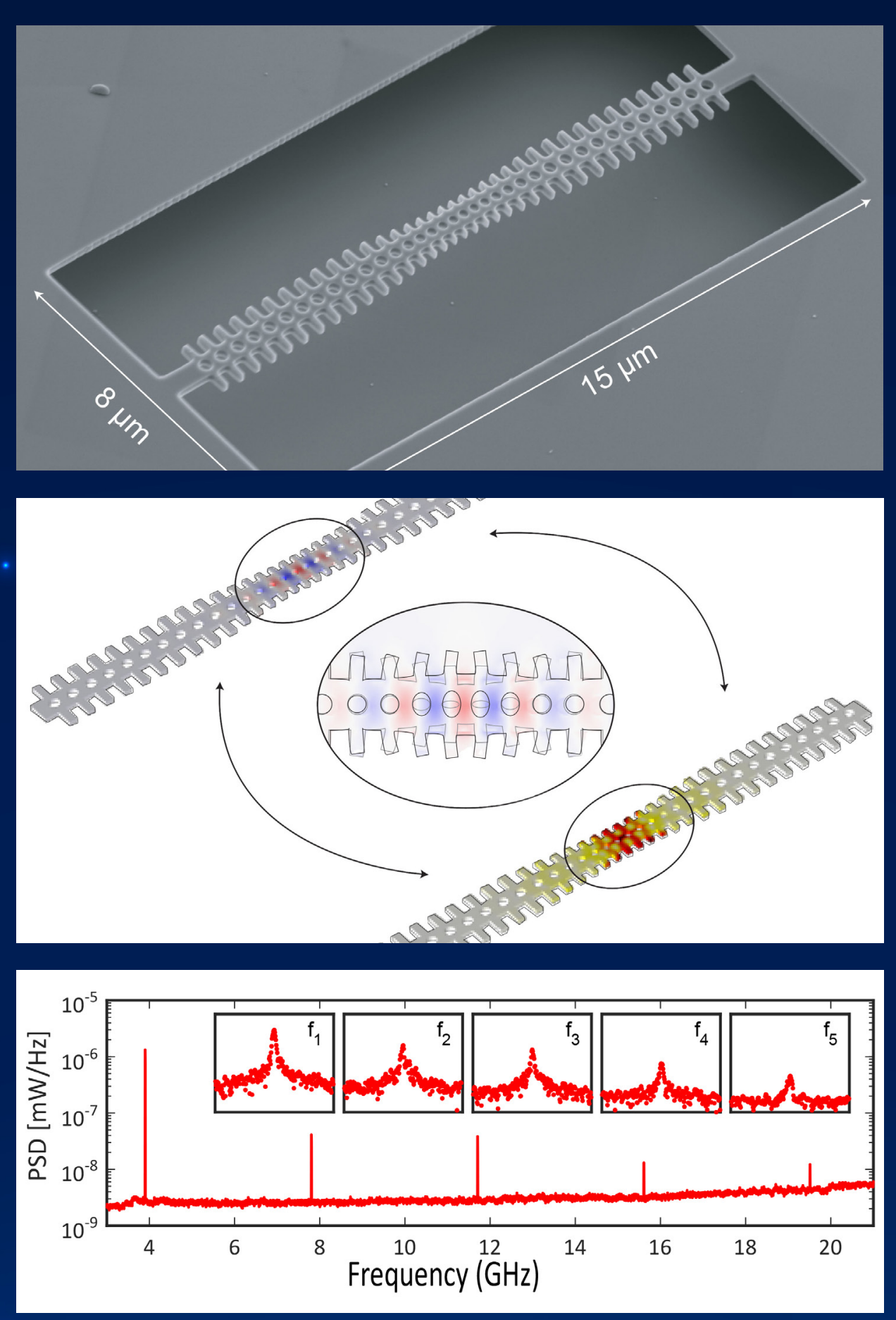

Figure 1: (a) Scanning electron microscopy image of the silicon optomechanical crystal cavity. (b) Optical (top) and mechanical (bottom) field profiles of the optomechanical system. The inset figure shows mechanical mode displacement. (c) Generated RF spectrum after photodetection of the OMO signal, 

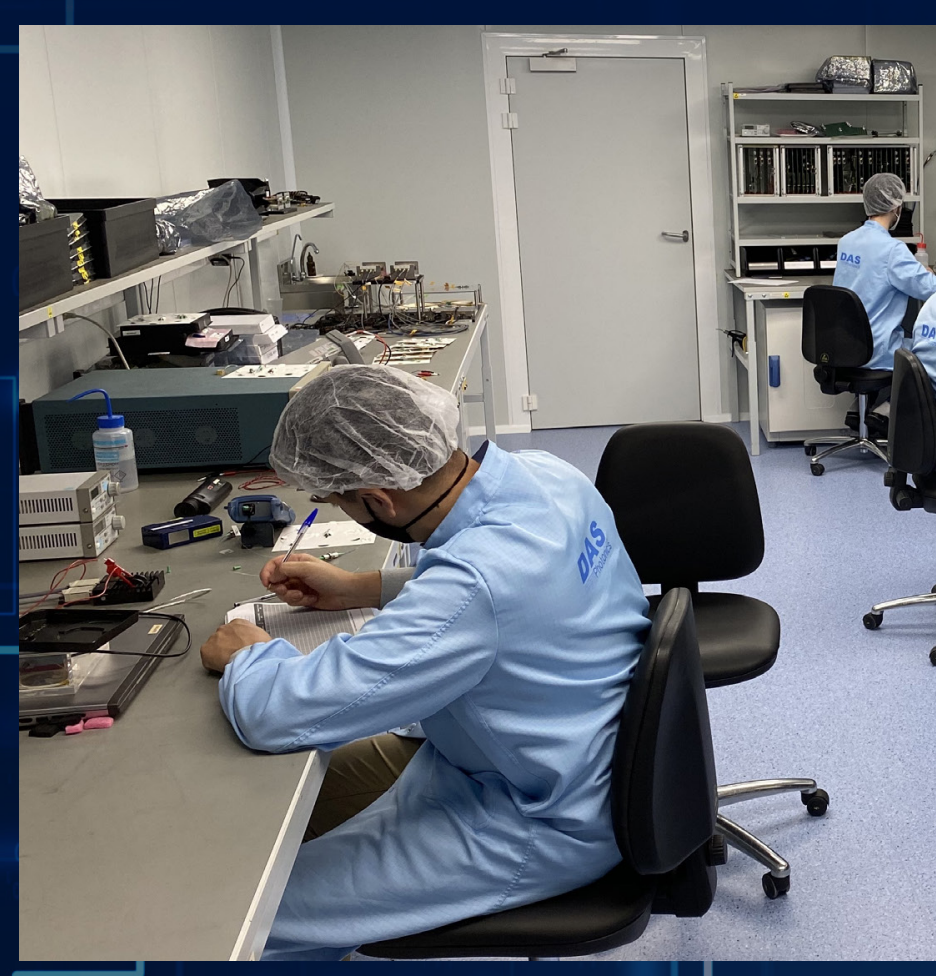

Figure 2: DAS Photonics clean-room to test devices for SATCOM applications. Indeed, this OMO has three key advantages:

i. it is fabricated in standard silicon technology, meaning that it can be potentially manufactured in large volumes at low cost as well as that it can be easily interconnected with electronics;

ii. it can be easily connected to optical fibres; and

iii. it is extremely compact and low作

The SIOMO project aims to turn a siliconphotonics-integrated $\mathrm{OMO}$, developed at the NTC (partner UPV), into a genuine economic innovation by addressing its technological transfer to the space sector via collaboration with the Spanish SME DAS Photonics. DAS Photonics is an experienced Spanish company providing high-end solutions based on its proprietary photonics technology for the most demanding applications worldwide.

To achieve this ambitious goal, SIOMO planned to test the developed OMO sing parameters for et al., 2021). This feature highlights its ATCOM) environments. To this end driven wireless access netwoton (like DAS's engineers assesed its practical $5 \mathrm{C}$ ), where the frequency conserion plicability on a real pace appliction process to adapt data strems to the est bed (see Figure 2) like in the context right frequency chenne is a key proces. of the previous OPTIMA proiect, where DAS Prous OPTima project, where Yet some improvements are still of the most important benefits of a necessary. The OMO needs to be photonic payload in SATCOM systems. packsed and con needs to be Measurements of the phese noise as well polarisation-maintaining fibre and its sthe frequency conversion efficiency interface with fibre improved to set f the OMO were carried out Results better power efficiency. All these steps onfirmed the potential of the new chnology in SATCOM applications.

particulin, using hamonics of Overall SIOMO has demonstrated the generated microwave tone the how a technology (silicon OM cavity) ighly-relevant X-band (between 8 developed in the framework of a 作 $12 \mathrm{GHz}$ is attainable. Therefore, fundamental-research project has the (SATCOM sytems) sIOMO aplication (he techno made it possible to bench hark this Noticeably, the SIOMO device acting in the market, leading to the elabortion imultaneouly as a photonic local of an industrialisation roadmap towards of fort ere it can be used to down- and up-convert out of the lab so that local tecillators in data frequency domain multiplexing (Mercadé made of silicon-technology OMOs.
References

Mercade, L., Griol, A., Navarro-Urrios, D. and Martínez, A. (2020) 'Microwave generation and frequer-
cy comb in a silicon optomechanic, 3535-3543. dio: 10.1515/nanoph-2020-0148. Mercadé, L., Morant, M., Griol, A., Llorente, R. and Martínez, A.(2021) 'Photonic frequency conversion
of OFDM microwave signalas in wavelength-scale optomechanical cavity', Laser \& Photonics Reviews,
15.

SIOMO video:

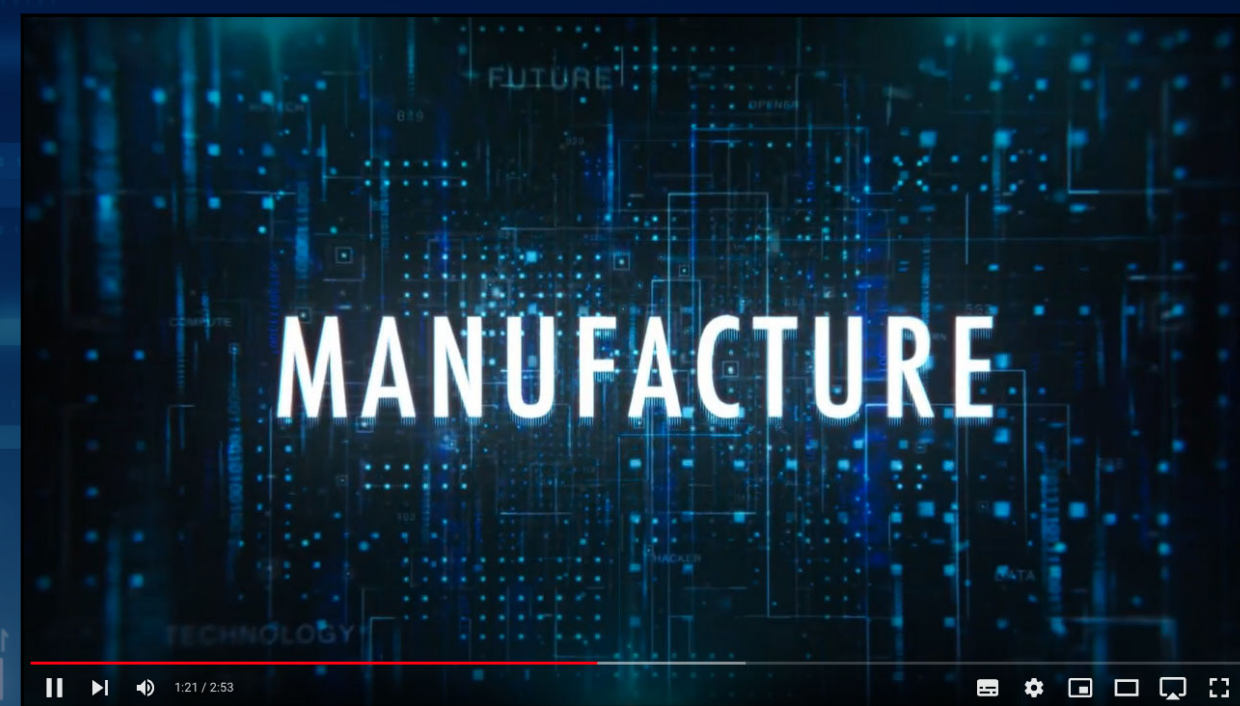

https://www.youtube.com/watch?v=y89sW/pJhfw\&t=79s

Websites:

NTC

https://ntc.webs.upv.es

DAS Photonics

https://www.dasphotonics.com $0 \quad 0$

SIOMO project

https://www.siomo-project.eu
PROJECT NAME

Sllicon Optomechanical

optoelectronic

Microwave Oscillator

SIOMO

PROJECT SUMMARY

SIOMO aims at turning a silicon-photonics optomechanical microwave oscillator
(OMO) into a genuine economic innovation by addressing its technological transfer to the space sector via the SME company DAS Photonics. To this end, the OMO has been
tested in a SATCOM test bed, showing its tested in a SATCOM test bed, showing
suitability to be used as a photonics-based suitability to be used as a photonics-based
local oscillator operating in the $\mathrm{X}$-band in satellites and aircrafts.

PROJECT LEAD PROFILE

Prof. Alejandro Martinez is leading the Plasmonics and Optomechanics group at Universitat Politècnica de València (Spain). His research interests include cavity optomechanics, plasmonics, nanoantennas and metamaterials and its integration into
silicon photonics chips. He has co-authored silicon photonics chips. He has co-authored
over 100 papers in international peerreviewed journals and holds eight patents. and more than 30 masters' theses.

\section{PROJECT PARTNERS}

Nanophotonics Technology Center at Universitat Politècnica de
DAS Photonics (Spain)

PROJECT CONTACTS

Prof. Alejandro Martínez

Camino de Vera s/n, Building 8F, 2nd floor, 46022 Valencia (Spain)

II +34 963877000 (Ext:88115)

$\triangle$ amartinez@ntc.upv.es

hisp://personales.upv.es/almarab/

@AMartinezUPV

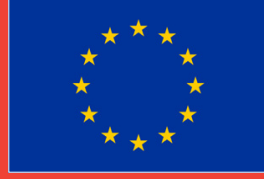

FUNDING

This project has received funding from the European Research Council (ERC) under the European Union's
Horizon 2020 research and innovation programme Horizon 2020 research and innovaton p 